\title{
Medication use and its impact on high-cost health care users among older adults: protocol for the population-based matched cohort HiCOSTT study
}

\author{
Justin Lee MD BScPhm, Sergei Muratov MD PhD, Jean-Eric Tarride PhD, J. Michael Paterson MSc, \\ Kednapa Thavorn PhD, Lawrence Mbuagbaw MD PhD, Tara Gomes PhD, Wayne Khuu MPH, \\ Hsien Seow PhD, Lehana Thabane PhD, Anne Holbrook MD PharmD
}

\section{Abstract}

Background: Health interventions and policies for high-cost health care users (HCUs) who are older adults need to be informed by a better understanding of their multimorbidity and medication use. This study aims to determine the financial contribution of medications to HCU expenditures and explore whether potentially inappropriate prescribing is associated with incident HCU development.

Methods: This is a protocol for a retrospective population-based matched cohort analysis of incident older adult HCUs (those with the highest $5 \%$ of costs and 66 years of age or older) in Ontario during fiscal year 2013. We will obtain person-level data for the index year and year before HCU status from health administrative databases and match each HCU to 3 non-HCUs based on age, sex and geographic location. Average annual medication costs (per patient) and the ratio of medication to total health care costs (at population level) will be examined over the HCU transition period and compared with non-HCUs. We will explore potential quality improvement areas for prescribing by analyzing chronic conditions and the use of medications with a strong evidence base for either clinical benefit or risk of harms outweighing benefits in older adults with these diagnoses. The relation between these medication classes and incident HCU status will be explored using logistic regression.

Interpretation: Using a matched cohort design and focusing on incident rather than prevalent HCUs, this protocol will explore our hypotheses that medications and the quality of their prescribing may be important triggers of HCU status and facilitate the identification of potential preventive clinical interventions or policies. Dissemination of results will occur via publications in peer-reviewed journals, presentations at conferences and academic settings, and knowledge translation activities with relevant health system and patient stakeholder groups. Study registration: Clinicaltrials.gov, no. NCT02815930

$\mathrm{H}$ igh-cost health care users (HCUs) are a small segment of the population that use disproportionate health care resources. In $2011,5 \%$ of individuals in Ontario, Canada, accounted for $65 \%$ ( $\$ 19.8$ billion) of the province's total measurable health care expenditures. ${ }^{1}$ These individuals are often characterized by frequent admission to hospital, comorbid mental illness, socioeconomic challenges and increased mortality risk. ${ }^{2}$ With health care costs expected to double in 20 years, the current approach to providing and financing health care for HCUs has been deemed unsustainable without major reforms. ${ }^{3,4}$ Similar findings have been reported internationally including those from the United States, United Kingdom and Australia. ${ }^{5-7}$

Despite increasing international scrutiny, the clinical epidemiology and economic impact of HCUs are not well understood. Most interventional studies have focused on case management, care coordination and disease management of high-risk or high-needs patients to prevent emergency department visits and admissions to hospital. ${ }^{2}$ However, it is unclear whether these interventions substantially improve clinical outcomes, decrease use of health care or reduce health care expenditures; studies of these interventions have had mixed and inconsistent results, and the overall quality of

Competing interests: Justin Lee reports a fellowship award from the Canadian Institutes of Health Research (CIHR) and studentship awards from the Ontario Drug Policy Research Network, the CIHR Drug Safety and Effectiveness Cross-Disciplinary Training Program, The Research Institute of St. Joe's Hamilton and St. Peter's/McMaster Chair in Aging, during the conduct of the study. No other competing interests were declared.

This article has been peer reviewed.

Correspondence to: Justin Lee, justin.lee@medportal.ca

CMAJ Open 2021. DOI:10.9778/cmajo.20190196 
evidence is low using the Grading of Recommendations Assessment, Development and Evaluation (GRADE) criteria. $^{2,8}$

Studies have also described the presence of both transient HCUs and persistent HCUs. Although there are transient HCUs who experience a time-limited acute health event and recover, an estimated $45 \%$ become persistent HCUs. ${ }^{9}$ The event may result in a transition to a new state of frailty or health management requirement that is challenging to reverse or stop. In both cases, the initial HCU transition might be preventable or delayed with earlier intervention.

Some of the primary challenges have been determining which patients, comorbidities, medications and health care services are most likely to benefit from intervention and identifying the key elements of a successful strategy. A recent analysis suggested that improved HCU management would require different tactics for different HCU subpopulations because of causes and solutions that vary by age and context. ${ }^{9}$

Older adults aged 65 years and older arguably represent the most important HCU subpopulation that needs to be targeted. They account for the largest proportion of HCUs, and this age group incurs the greatest median health care expenditures among HCUs. ${ }^{9}, 10$ Among older adults in Ontario, HCUs with the top $5 \%$ of costs account for $44 \%$ of total health care expenditures in that age group. We have previously described their unplanned admissions to hospital and the incremental and regional variation of their health care costs. ${ }^{11-13}$ However, the impact of medication costs and the quality of prescribing on HCU development remains unclear. The prevalence of potentially inappropriate prescribing is reported to be $21 \%-79 \%$ in the general older adult population, and it is likely higher in the older adult HCU population given higher rates of polypharmacy. ${ }^{14,15}$

Planning interventions and developing effective health policy for older adult HCUs requires a deeper understanding of their demographic characteristics, comorbidities, medications, health care providers, and health service use and associated costs compared with those of non-HCUs. To address these knowledge gaps, we will conduct the High-cost User Characterization of Ontario's Seniors' Medication Use and Health care Utilization (HiCOSTT) study.

By exploring the patterns of health service and medication use during the transition period from non-HCU to HCU, we seek to identify opportunities to improve the quality of care and prevent HCU development. This may include the development of new health-related policies or targets for intervention, such as specific high-risk patients suited for more intensive transitional care programs or high-cost drugs suited for reference-based drug pricing reimbursement.

This study's primary objectives are to determine the relative financial contribution of prescription medications to total health care expenditures in incident older adult HCUs, and to explore whether suboptimal or potentially inappropriate prescribing is associated with increased health care expenditures and incident $\mathrm{HCU}$ development in older adults. The secondary objective is to characterize how the clinical profiles and prognoses of older adult HCUs differ from nonHCUs by describing their sociodemographic characteristics, comorbidities, health service use, medication use, clinical outcomes and health care expenditures.

\section{Methods}

\section{Study design}

This is a retrospective population-based matched cohort study protocol that is registered with clinicaltrials.gov (NCT02815930). We are concurrently using a case-control study design to discriminate the impact of specific medication classes on the development of HCU status. We report this protocol with guidance from the Reporting of Studies Conducted Using Observational Routinely Collected Health Data Statement for Pharmacoepidemiology (RECORD-PE) checklist. ${ }^{16}$ Table 1 describes the main research questions, hypotheses and outcome measures.

\section{Observational periods}

The observational study period will run from Apr. 1, 2010, to Mar. 31, 2014. The accrual period to ascertain exposure (i.e., HCU status) will extend from the index date of Apr. 1, 2013, to the maximum follow-up date of Mar. 31, 2014. This will allow for a 1-year look-back period to determine incident HCU, baseline demographic information, and medication and health services use data before HCU status as well as a 3-year look-back period to determine comorbidities.

\section{Participants}

This study will examine a population-based cohort of incident older adult HCUs aged 66 years or older with annual total health care expenditures within the top $5 \%$ threshold of all residents in Ontario. ${ }^{9}$ The top $5 \%$ financial threshold will be determined using costing algorithms of ICES person-level health care use, which have previously been described in detail. ${ }^{17}$ Total health care use expenditures for each eligible Ontario resident in the fiscal year will be calculated. We will then sort individuals by expenditures to identify the top $5 \%$ of HCUs.

We will include patients if they were Ontarians registered in the Ontario Health Insurance Plan (OHIP) Registered Persons Database during the accrual period and aged 66 years or older on the index date of Apr. 1, 2013. Patients will be excluded if they do not have a valid OHIP number or died on or before the index date.

Study cohort selection is shown in Figure 1. The prevalent HCU cohort will be defined as those patients who had annual total health care expenditures equal to or greater than the top $5 \%$ financial threshold for fiscal year 2013. The incident HCU cohort will be defined as those patients in the prevalent HCU cohort whose annual total health care expenditures in fiscal year 2012 did not meet the top aforementioned 5\% financial threshold. The non-HCU cohort will be defined as those patients whose annual total health care expenditures were less than the top 5\% financial threshold in both fiscal year 2012 and fiscal year 2013. 
Table 1: Main research questions, hypotheses and outcome measures

Main research questions Hypotheses Study design main outcome measures

\section{Primary}

1. What is the relative financial contribution of prescription medications to incident $\mathrm{HCU}$ expenditures and how do they compare with non-HCUs?

\section{- Prescription medication} costs will rank within the top 3 cost categories of $\mathrm{HCU}$ expenditures

- Average medication costs (on an individual level) and the proportion of costs attributable to medications (at a population level relative to total costs) are different in HCUs and non-HCUs

- In a subset of incident HCUs, prescription medication costs alone will be greater than the financial threshold for HCU status

2. What is the relative clinical contribution of prescription medications to incident $\mathrm{HCU}$ status? (i.e., Does the quality of medications prescribed and used contribute to differences in health care costs and $\mathrm{HCU}$ development?)
- The use of "high quality" medication classes (i.e., those with a strong evidence base for primary or secondary prevention selected a priori for analysis) will be associated with a decreased odds of incident HCU status

- The use of "potentially inappropriate or high risk" medications selected a priori for analysis will be associated with an increased odds of incident HCU status

- The use of "high cost (per unit)" medications for recognized indications will be associated with an increased odds of incident HCU status
Retrospective matched cohort analysis (HCU status treated as an "exposure")

Case-control analysis ( $\mathrm{HCU}$ status treated as an outcome)
- Annual total prescription medication costs (COprimary outcome)

- Annual drug cost to total health care expenditure ratio (co-primary outcome)

- Frequency of patient cases in which annual drug costs alone exceeds health expenditure threshold for HCU status - Odds ratio of incident HCU
status
Retrospective matched cohort analysis (HCU status treated as an "exposure")
- Number of Johns Hopkins Adjusted Diagnosis Groups and Expanded Diagnosis Clusters

- Number of unique prescription drug classes dispensed

- All-cause mortality rate

- All-cause hospital admission rate admissions com non-HCUs

4. What is the prevalent use of prescription medication classes with a strong evidence base for primary or secondary prevention of complications associated with the most common chronic conditions?

\section{- In the pre-HCU year, the} prevalent use of "high quality" prescription medication classes will be lower in HCUs compared with non-HCUs with the relevant associated indications
Retrospective matched cohort analysis (HCU status treated as an "exposure")
- Prevalent use of "high quality" medication classes selected a priori for analysis

- Prevalence of relevant chronic condition based on John Hopkins Expanded Diagnosis Clusters and chart-validated ICES chronic disease cohorts

Note: $\mathrm{HCU}=$ high-cost health care user 


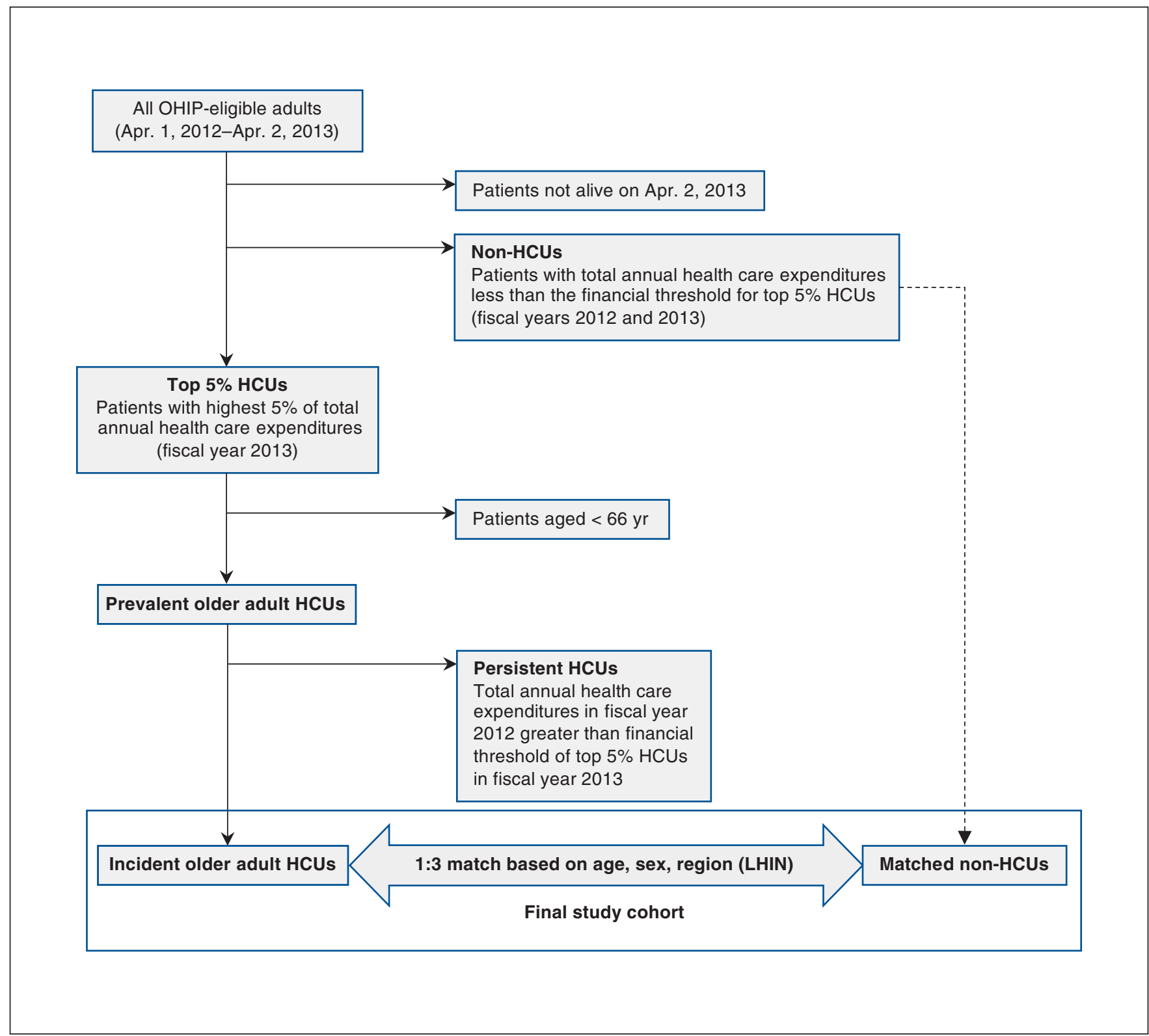

Figure 1: Study cohort selection. Note: HCUs = high-cost health care users, LHIN = Local Health Integration Network, OHIP = Ontario Health Insurance Plan.

We will match the incident HCU cohort without replacement to a cohort of non-HCUs using a 3:1 matching ratio (non-HCU to HCU) based on age at cohort entry $( \pm 1 \mathrm{mo}$ ), sex and geographic location for comparative analysis. The geographic location will be based on Local Health Integration Networks, which are the health authorities responsible for regional administration of public health care services in Ontario.

We will create the cohorts in this fashion to focus on incident HCUs (rather than those persisting or transitioning out of HCU status), to include as many incident HCUs as possible to look for important cohort and subgroup characteristics (i.e., comorbidities, medication use and health care use, and to minimize confounding because of age, gender and geogra- phy). The 3:1 matching ratio was selected to increase our statistical efficiency. ${ }^{18}$

\section{Databases used}

We will obtain patient-level data from ICES, which holds linked health administrative databases for Ontario's publicly funded health care services using patient-specific, coded identifiers. This study will use 19 health administrative databases (described in Table 2) to track all health service encounters such as physician billings, hospital admissions and prescription drugs. More detailed descriptions of the individual variables contained in these databases can be found in the ICES data dictionary. ${ }^{23}$ All of these data sets are held securely at ICES and analyzed in linked, coded form using a patient- 


\begin{tabular}{|c|c|c|}
\hline Health administrative database & Description & Database variables or data used \\
\hline $\begin{array}{l}\text { Ontario Registered Persons } \\
\text { Database (RPDB) }\end{array}$ & $\begin{array}{l}\text { The RPDB records vital statistics, including date of } \\
\text { death. }\end{array}$ & $\begin{array}{l}\text { - Date of death } \\
\text { - Rurality Index Ontario Score } \\
\text { - Age } \\
\text { - Sex }\end{array}$ \\
\hline $\begin{array}{l}\text { Immigration, Refugees and } \\
\text { Citizenship Canada } \\
\text { Permanent Resident Database }\end{array}$ & $\begin{array}{l}\text { The CIC database contains landing records for every } \\
\text { permanent legal immigrant to Canada who arrived } \\
\text { from } 1985 \text { onward. }\end{array}$ & - Date of landing or immigration \\
\hline $\begin{array}{l}\text { Local Health Integration } \\
\text { Network (LHIN) database }\end{array}$ & $\begin{array}{l}\text { The LHIN database contains records of the health } \\
\text { authorities responsible for the regional } \\
\text { administration of public health care services in the } \\
\text { province. }\end{array}$ & $\begin{array}{l}\text { - Geographic location of residence of included } \\
\text { patients }\end{array}$ \\
\hline $\begin{array}{l}\text { Ontario Drug Benefit (ODB) } \\
\text { database }\end{array}$ & $\begin{array}{l}\text { The ODB database contains highly accurate } \\
\text { records for outpatient prescriptions dispensed to } \\
\text { patients aged } 65 \text { yr or older (with error rates } \\
\text { reported to be }<1 \%) .{ }^{19}\end{array}$ & $\begin{array}{l}\text { - Prescription drug fill dates and costs } \\
\text { - Long-term care indicator }\end{array}$ \\
\hline $\begin{array}{l}\text { Canadian Institute for Health } \\
\text { Information Discharge Abstract } \\
\text { Database (CIHI-DAD) }\end{array}$ & $\begin{array}{l}\text { The CIHI-DAD contains validated patient-level } \\
\text { demographic, diagnostic, procedural and treatment } \\
\text { information on all acute- and long-term-care hospital } \\
\text { admissions. }\end{array}$ & $\begin{array}{l}\text { - ICD-10 codes for hospital discharge } \\
\text { diagnoses and Johns Hopkins ACGs and } \\
\text { EDCs } \\
\text { - Hospital admissions (elective and urgent) } \\
\text { and costs }\end{array}$ \\
\hline $\begin{array}{l}\text { CIHI - National Ambulatory } \\
\text { Care Reporting System } \\
\text { (NACRS) database }\end{array}$ & $\begin{array}{l}\text { The CIHI-NACRS database contains patient-level } \\
\text { demographic, diagnostic, procedural and treatment } \\
\text { information for all hospital-and community-based } \\
\text { ambulatory care including day surgery, outpatient and } \\
\text { community-based clinics and emergency } \\
\text { departments. }\end{array}$ & $\begin{array}{l}\text { - ICD-10 codes for hospital discharge } \\
\text { diagnoses and Johns Hopkins ACGs and } \\
\text { EDCs } \\
\text { - Visits and costs }\end{array}$ \\
\hline $\begin{array}{l}\text { ClHI — Same Day Surgery } \\
\text { (SDS) }\end{array}$ & $\begin{array}{l}\text { The CIHI-SDS contains patient-level demographic, } \\
\text { diagnostic, procedural and treatment information for } \\
\text { all day surgeries. }\end{array}$ & $\begin{array}{l}\text { - ICD-10 codes for hospital discharge } \\
\text { diagnoses and Johns Hopkins ACGs and } \\
\text { EDCs } \\
\text { - Visits and costs }\end{array}$ \\
\hline $\begin{array}{l}\text { CIHI — National Rehabilitation } \\
\text { Reporting System (NRS) }\end{array}$ & $\begin{array}{l}\text { The CIHI-NRS contains patient-level demographic, } \\
\text { diagnostic, procedural and treatment information from } \\
\text { participating adult inpatient rehabilitation facilities and } \\
\text { programs. }\end{array}$ & - Visits and costs \\
\hline $\begin{array}{l}\text { Ontario Home Care Database } \\
\text { (HCD) }\end{array}$ & $\begin{array}{l}\text { The Ontario HCD contains patient-level demographic, } \\
\text { diagnostic, procedural and treatment information or all } \\
\text { home care visits. }\end{array}$ & $\begin{array}{l}\text { - Home care visits } \\
\text { - Type of home care service provided } \\
\text { - Visits and costs }\end{array}$ \\
\hline $\begin{array}{l}\text { Ontario Continuing Care } \\
\text { Reporting System (CCRS) }\end{array}$ & $\begin{array}{l}\text { The Ontario CCRS contains demographic, clinical, } \\
\text { functional and resource use information or patients } \\
\text { receiving continuing care services in hospitals or } \\
\text { long-term care homes in Canada. }\end{array}$ & - Visits and costs \\
\hline $\begin{array}{l}\text { Ontario Mental Health } \\
\text { Reporting System (OMHRS) } \\
\text { database }\end{array}$ & $\begin{array}{l}\text { The OMHRS database contains patient-level } \\
\text { demographic, diagnostic, procedural and treatment } \\
\text { information for all psychiatric facility visits. }\end{array}$ & - Visits and costs \\
\hline $\begin{array}{l}\text { ICES Physician Database } \\
\text { (IPDB) }\end{array}$ & $\begin{array}{l}\text { The IPDB reports prescriber and specialist referral } \\
\text { and billing data in Ontario. The physician } \\
\text { demographic data are validated against the } \\
\text { Ontario Physician Human Resource Data Centre } \\
\text { database. }\end{array}$ & - Visits to primary care and specialists \\
\hline $\begin{array}{l}\text { Ontario Health Insurance Plan } \\
\text { database (OHIP) }\end{array}$ & $\begin{array}{l}\text { The OHIP database includes health claims for } \\
\text { physician services. }\end{array}$ & $\begin{array}{l}\text { - ICD-10 codes for hospital discharge } \\
\text { diagnoses and Johns Hopkins ACGs and } \\
\text { EDCs } \\
\text { - All health service visits and costs }\end{array}$ \\
\hline $\begin{array}{l}\text { Client Agency Program } \\
\text { Enrolment (CAPE) database }\end{array}$ & $\begin{array}{l}\text { The CAPE database contains enrolment information } \\
\text { of an individual in a program with a specific } \\
\text { practitioner and group. }\end{array}$ & $\begin{array}{l}\text { - Primary care practitioner reimbursement } \\
\text { model of included patients }\end{array}$ \\
\hline
\end{tabular}


Table 2 (part 2 of 2): Health administrative databases

\begin{tabular}{|c|c|c|}
\hline Health administrative database & Description & Database variables or data used \\
\hline $\begin{array}{l}\text { ICES-derived cohorts: } \\
\text { - Congestive Heart Failure } \\
\text { database } \\
\text { - Chronic Obstructive } \\
\text { Pulmonary Disease } \\
\text { database } \\
\text { - Ontario Crohn's and Colitis } \\
\text { Cohort dataset } \\
\text { - Ontario Diabetes Dataset } \\
\text { - Ontario Myocardial Infarction } \\
\text { Database Dataset } \\
\text { - Ontario Rheumatoid Arthritis } \\
\text { Dataset }\end{array}$ & $\begin{array}{l}\text { The ICES-derived cohorts are chart-validated cohorts } \\
\text { of patients with specific diseases and conditions. } \\
\text { These cohorts are created using health administrative } \\
\text { case definitions that link hospital inpatient and } \\
\text { outpatient care, physician claims and drug benefits } \\
\text { data over time using an anonymous unique } \\
\text { identifier. }{ }^{20} \text { The case definitions are validated by } \\
\text { clinical scientists at ICES using data collected directly } \\
\text { from reviews of medical charts in the community. For } \\
\text { example, the diabetes and hypertension definitions } \\
\text { have a positive predictive value of } 80 \% \text { and } 87 \% \text {, } \\
\text { respectively. }{ }^{21,22}\end{array}$ & - Comorbidities of included patients \\
\hline
\end{tabular}

specific identifier. ICES is permitted to receive and use personal health information through a special designation under Ontario's Personal Health Information Protection Act (PHIPA). This designation is maintained through a tri-annual review and approval process undertaken by the Office of the Information and Privacy Commissioner of Ontario, which is described at https://www.ices.on.ca/Data-and-Privacy/ Privacy-at-ICES.

\section{Study outcomes}

Study outcomes are summarized in Table 1. This study's coprimary outcomes are the between-group (incident HCU versus non-HCU) differences of the following in the 1-year period after the index date: the annual total prescription medication expenditures per patient and the annual drug cost to total health care expenditure ratio. The study's secondary clinical outcomes are the between-group differences in the 1-year mortality and rates of hospital admission.

We will determine mortality using records from the OHIP Registered Persons Database. The rate of hospital admissions will be determined by dividing the frequency of hospital admissions by the total number of days for which the patients were not admitted (i.e., days at risk) during the given year. A "hospital admission" will be defined as each unique "episode of care." An episode of care links a series of hospital admissions to prevent interhospital transfers being counted as a readmission.

\section{Statistical analysis}

We will present descriptive statistics for baseline clinical and demographic characteristics of both cohorts and for study outcomes. Standardized differences will be used to compare the distribution of baseline covariates between groups for the matched cohort. We will interpret a standardized difference greater than 0.1 as a meaningful difference. ${ }^{24}$ Missing data for any covariates will be reported as separate categories. Since our sample size is expected to be large, we will use pairwise deletion unless the proportion of missing data for any variable is greater than $5 \%$ and an independent statistician suggests multiple imputation..$^{25}$
Baseline patient characteristics will be obtained for the index date including sociodemographic characteristics (e.g., age, gender, income quintile, social assistance recipient and immigration status), geographic distribution (e.g., health region and rurality) and primary care exposure. The Rurality Index of Ontario score will be used to define urban, suburban and rural residence using the Rurality Index of Ontario scores of less than 10,10-39 and $\geq 40$, respectively. Patients will be considered recent immigrants if they landed in Canada less than 15 years from the index date according to the Immigration, Refugees and Citizenship Canada Permanent Resident database. We will characterize patients' primary care exposure based on whether they have an identified provider in the Client Agency Enrolment Program database and the type of payment model for that provider (e.g., feefor-service v. capitation).

The baseline comorbidity burden of each cohort on the index date will be summarized by using John Hopkins Adjusted Clinical Groups (ACGs) derived from the Johns Hopkins ACG System version 10, using hospital admission and ambulatory care encounter data over 3 years preceding the index date. The ACG system assigns International Statistical Classification of Diseases and Related Health Problems, 10th Revision (ICD-10) diagnosis codes from inpatient and ambulatory health administrative data to 1 of 32 diagnosis clusters known as Aggregated Diagnosis Groups (ADGs). A previous retrospective cohort study in Ontario found that ADGs accurately predict 1-year mortality in a general population cohort. ${ }^{26}$ Comorbidities will be profiled by focusing on the most prevalent, clinically important or economically important disease states identified by John Hopkins Expanded Diagnosis Clusters (EDCs). Based on the ACG System, EDCs are groupings of diagnostic codes that describe the same or related condition. When available, the chart-validated chronic disease indicators (e.g., chronic obstructive pulmonary disease and congestive heart failure) developed by ICES will be preferentially used for any diagnoses that have complementary EDCs.

In the incident year and year before becoming an HCU, we will delineate drug and health care use of both cohorts to 
compare how health care resources are being used by older adult HCUs and non-HCUs. This will include annual usage rates of emergency departments, hospitals, physician visits and home care. We will determine reasons for hospital admission using discharge abstract ICD codes. Health care expenditures will also be broken down into key components (e.g., hospital admission, long-term care, home care and drugs) as per the ICES costing algorithm. ${ }^{17}$ Similar analyses will be conducted in the year of incident HCU designation and for non-HCUs.

Identification of the most commonly used therapeutic medication classes and the most common reasons for hospital admissions will be used to hypothesize care management issues that may be contributing to HCU development. Previous studies have highlighted the existence of important prescribing practice gaps, and the use of potential prescribing omissions and inappropriate medication use as quality indicators of pharmacologic care in older adults. ${ }^{27-29}$ Two such examples include suboptimal prescribing and use of statins $(<30 \%)$ and bisphosphonates $(<50 \%)$ in those with a history of cerebrovascular disease and osteoporosis, respectively. ${ }^{27,28}$ In a similar fashion, we will compare the prevalent use of specific medication classes with the relevant diagnoses of patients within these cohorts to explore potential quality improvement areas for prescribing and the financial implications of current drug use by HCUs v. non-HCUs and health care usage prev. post-HCU designation.

We have selected medication classes a priori for analysis based on 3 considerations: those with a strong evidence base for the prevention of complications in common, high-priority disease states in older adults ("high-quality" medications); those with a strong evidence base for harms outweighing potential benefits or those commonly targeted for deprescribing in older adults because of potential inappropriateness (e.g., sedative-hypnotics, antipsychotics and opioids; "highrisk" medications); and those known to be high-cost (per unit) medications (Table 3). We chose these classes as high-priority medications based on review of expert consensus guidelines on potentially inappropriate prescribing in older adults (i.e., START/STOPP and Beers Criteria), Canadian deprescribing guidelines and previous studies of potentially inappropriate prescribing and high-cost medication use in Ontario. ${ }^{30-35}$ Medication use will be defined as the occurrence of at least 1 prescription claim during the study period.

Using a case-control study design with HCU development as the outcome (i.e., incident HCUs are the cases and the matched non-HCUs are the controls), we will conduct logistic regression to explore the relation between the use of these medications and incident HCU status. Each medication class will be analyzed independently in a series of analyses. To minimize potential bias from confounding by indication and severity of illness, we will adjust estimates for age, sex, number of comorbidities, number of major John Hopkins ADGs and the most common conditions for which the drugs are indicated. As a variation of the traditional dose-response relation in the Bradford-Hill criteria for potential causal association, we will incorporate duration of medication use (within the study window) into the analysis to explore any associations with HCU transition based on increasing exposure to the drugs. ${ }^{36}$

We will use these data to explore whether more in-depth analysis is required (i.e., are there signals that potential drugrelated interventions before HCU designation could be implemented to avoid critical health care events, minimize drug-related expenditures or prevent HCU status?)

Table 3: Medication classes of interest for patient-level analysis

\begin{tabular}{|c|c|c|}
\hline High-quality medication use & $\begin{array}{l}\text { Potentially inappropriate or } \\
\text { high-risk medication use }\end{array}$ & High-cost drug use \\
\hline $\begin{array}{l}\text { - Statins } \\
\text { - } \beta \text {-blockers } \\
\text { - ACE inhibitors } \\
\text { - Angiotensin receptor blockers } \\
\text { - Antiplatelet agents } \\
\text { (e.g., ASA, adenosine } \\
\text { diphosphosphate inhibitors, platelet } \\
\text { aggregation inhibitors) } \\
\text { - Anticoagulants } \\
\text { - Vitamin K antagonists } \\
\text { - DOACs - factor Xa inhibitors } \\
\text { - DOACs - direct thrombin inhibitors } \\
\text { - Bisphosphonates } \\
\text { - Bone calcium regulators } \\
\text { (e.g., denosumab) } \\
\text { - Bronchodilator and anti-inflammatory } \\
\text { combination inhalers } \\
\text { - Long-acting anti-cholinergic inhalers } \\
\text { (e.g., tiotropium) }\end{array}$ & $\begin{array}{l}\text { - Proton pump inhibitors } \\
\text { - Benzodiazepines } \\
\text { - Narcotics (opiate agonists) } \\
\text { - Antipsychotics } \\
\text { - NSAIDs (non-ASA) } \\
\text { - Digitalis preparations (digoxin) }\end{array}$ & $\begin{array}{l}\text { - Immunosuppressive agents (e.g., mycophenolic acid, } \\
\text { natalizumab, sirolimus, tacrolimus, thalidomide) } \\
\text { - Antineoplastic agents (e.g., tocilizumab, imatinib, } \\
\text { dasatinib) } \\
\text { - Ophthalmologics (e.g., ranibizumab) } \\
\text { - Biologic response modifying agents (e.g., adalimumab, } \\
\text { aldesleukin, certolizumab, etanercept, glatiramer, } \\
\text { golimumab, infliximab, interferons, levamisole) }\end{array}$ \\
\hline
\end{tabular}




\section{Ethics approval}

This study has been approved by the Hamilton Integrated Research Ethics Board (No. 1715-C).

\section{Interpretation}

Comparative analysis of drug and health care use and expenditures during the HCU transition period will help identify key contributors to HCU development. We expect to find common groups of diagnoses, medications and clinical outcomes among older adult HCUs. Some of these conditions may be amenable to cost-effective preventive management strategies or less expensive but equally effective medication therapies. In particular, data from this study will describe the degree to which drugs costs contribute to overall health care costs in HCUs, confirm whether or not prescription drug costs rank within the top 3 cost categories of HCU expenditures, describe how medication and health care expenditures change with HCU status, and explore whether there are possible quality improvement areas for prescribing among older adult HCUs. This may facilitate the identification of potential high-yield areas for targeted clinical or medication management interventions to prevent HCU development, provision of data to support health policies for HCUs in Ontario and areas requiring further study.

This population-based matched cohort analysis will provide important data about older adult HCUs and their medication use. Use of linked health data in a large population of older adults ( $n=5352983$ ) provides a unique opportunity to compare and contrast the characteristics of HCUs to their non-HCU counterparts. ${ }^{37}$ The focus on incident rather than prevalent HCUs allows for the identification of potential triggers of HCU status and exploration of potential preventative interventions. The large estimated cohort size makes it possible to study predictors of high-cost health care use with statistical efficiency and examine their impact on clinical outcomes in a way that transcends differences in local practice patterns. Medication records from the provincial drug plan provide data that is generally more accurate than self-recorded information on drug use.

The results of this study will be shared via peer-reviewed publications and presentations at provincial, national and international conferences. Knowledge translation activities will include information exchange and policy recommendations to local and national health system and patient stakeholder groups.

\section{Limitations}

This study is subject to the limitations and residual confounding inherent in observational data from health administrative databases. It is not possible to guarantee that patients were adherent to dispensed medications nor that they were used as prescribed. Our ability to account for the duration of medication exposure is limited to 2 years of available prescription data. This may affect our ability to detect associations related to the timing of medication initiation and HCU transition.
Comorbidity and disease data are reliant on the use of hospital discharge diagnosis data using ICD and billing codes. Although the quality of discharge coding is generally good, we do not know the accuracy of every diagnosis listed on a discharge abstract. ${ }^{38}$ In addition, physicians are permitted only 1 diagnosis code for each office encounter. This means that acute problems often take precedence over long-term ones, which can serve to underrepresent the prevalence of chronic disease.

High-cost health care users and non-HCUs are being identified based on costs of health care use accrued during a fiscal year. Some patients (including those in their last year of life) may be classified differently using an alternative method depending on when they accrued costs or died.

\section{Conclusion}

This study will determine the relative contribution of medications to HCU expenditures and explore whether the quality of prescribing and medication use may be contributing to suboptimal clinical outcomes and their high-cost use. By identifying key contributors to HCU status, we will help clinicians, administrators and policy-makers determine which patients, diseases, drugs and expenses could benefit from intervention. If modest improvements in prescribing and medication use can be achieved, then there will likely be substantial health care savings that could be reinvested to fund better care for these high-risk, high-cost older adults.

\section{References}

1. Blair A, Baigent L. High cost users. Toronto: Ministry of Health and LongTerm Care; 2013.

2. Lee JY, Muratov S, Tarride J-E, et al. Managing high-cost healthcare users: the international search for effective evidence-supported strategies. $7 \mathrm{Am}$ Geriatr Soc 2018;66:1002-8.

3. Ontario population projections update, 2012-2036. Queen's Printer for Ontario; updated 2019 Oct. 1. Available: www.fin.gov.on.ca/en/economy/ demographics/projections/ (accessed 2014 Oct. 12).

4. Fiscal sustainability of health systems: bridging health and finance perspectives. Paris: Organisation for Economic Co-operation and Development; 2015.

5. Calver J, Brameld KJ, Preen DB, et al. High-cost users of hospital beds in Western Australia: a population-based record linkage study. Med $\mathcal{F}$ Aust 2006; $184: 393-7$

6. The concentration of health care spending [NIHCM Foundation data brief]. Washington (DC): National Institute for Health Care Management; 2012.

7. Bardsley M, Lewis G. Reflections from the NHS in England. Healthc Pap 2014;14:26-30, discussion 58-60.

8. Guyatt G, Oxman AD, Akl EA, et al. GRADE guidelines: 1. Introduction GRADE evidence profiles and summary of findings tables. 7 Clin Epidemiol 2011;64:383-94.

9. Wodchis WP, Austin PC, Henry DA. A 3-year study of high-cost users of health care. CMAf 2016;188:182-8.

10. Wodchis WP. The concentration of health care spending: little ado (yet) about much (money). In: Proceedings of the Canadian Association for Health Services and Policy Research conference; Montreal; 2012 May 30. Available: www. longwoods.com/blog/the-concentration-of-health-care-spending-little-ado-yet -about-much-money/ (accessed 2015 Oct. 1).

11. Muratov S, Lee J, Holbrook A, et al. Unplanned index hospital admissions among new older high-cost health care users in Ontario: a population-based matched cohort study. CMA7 Open 2019;7:E537-45.

12. Muratov S, Lee J, Holbrook A, et al. Incremental healthcare utilisation and costs among new senior high-cost users in Ontario, Canada: a retrospective matched cohort study. BMF Open 2019;9:e028637.

13. Muratov S, Lee J, Holbrook A, et al. Regional variation in healthcare spending and mortality among senior high-cost healthcare users in Ontario, Canada: a retrospective matched cohort study. BMC Geriatr 2018;18:262.

14. Hill-Taylor B, Sketris I, Hayden J, et al. Application of the STOPP/START criteria: a systematic review of the prevalence of potentially inappropriate prescribing in older adults, and evidence of clinical, humanistic and economic impact. 7 Clin Pharm Ther 2013;38:360-72. 
15. Weng M-C, Tsai C-F, Sheu K-L, et al. The impact of number of drugs prescribed on the risk of potentially inappropriate medication among outpatient older adults with chronic diseases. Q7M 2013;106:1009-15.

16. Langan SM, Schmidt SA, Wing K, et al. The reporting of studies conducted using observational routinely collected health data statement for pharmacoepidemiology (RECORD-PE). BM7 2018;363:k3532.

17. Wodchis WP, Bushmeneva K, Nikitovic M, et al. Guidelines on person-level costing using administrative databases in Ontario. Working Paper Series Vol. 1. Toronto: Health System Performance Research Network; 2013:1-71.

18. Breslow NE, Day NE. Statistical Methods in Cancer Research: Volume I - The Analysis of Case-Control Studies. IARC Scientific Publication No. 32. Lyon (France): International Agency for Research on Cancer; 1980. p. 169.

19. Levy AR, O'Brien BJ, Sellors C, et al. Coding accuracy of administrative drug claims in the Ontario Drug Benefit database. Can 7 Clin Pharmacol 2003;10:67-71.

20. Iron K, Lu H, Manuel D, et al. Using linked health administrative data to assess the clinical and healthcare system impact of chronic diseases in Ontario. Healthc Q 2011;14:23-7.

21. Hux JE, Ivis F, Flintoft V, et al. Diabetes in Ontario: Determination of prevalence and incidence using a validated administrative data algorithm. Diabetes Care 2002;25:512-6.

22. Tu K, Campbell NR, Chen Z-L, et al. Accuracy of administrative databases in identifying patients with hypertension. Open Med 2007;1:e18-26.

23. ICES Data Dictionary. Available: https://datadictionary.ices.on.ca/ (accessed 2020 Apr. 1)

24. Austin P. Using the standardized difference to compare the prevalence of a binary variable between two groups in observational research. Commun Stat Simul Comput 2009;38:1228-34.

25. Cheema JR. Some general guidelines for choosing missing data handling methods in educational research. 7 Mod Appl Stat Methods 2014;13.

26. Austin PC, van Walraven C, Wodchis WP, et al. Using the Johns Hopkins Aggregated Diagnosis Groups (ADGs) to predict mortality in a general adult population cohort in Ontario, Canada. Med Care 2011;49:932-9.

27. Brown F, Singer A, Katz A, et al. Statin-prescribing trends for primary and secondary prevention of cardiovascular disease. Can Fam Pbysician 2017; $63: \mathrm{e} 495-503$

28. Burden AM, Paterson JM, Solomon DH, et al. Bisphosphonate prescribing, persistence and cumulative exposure in Ontario, Canada. Osteoporos Int 2012;23:1075-82.

29. Higashi T, Shekelle PG, Solomon DH, et al. The quality of pharmacologic care for vulnerable older patients. Ann Intern Med 2004;140:714-20.

30. O'Mahony D, O'Sullivan D, Byrne S, et al. STOPP/START criteria for potentially inappropriate prescribing in older people: version 2 [published erratum in Age Ageing 2018;47:489]. Age Ageing 2015;44:213-8.

31. Deprescribing guidelines and algorithms. Bruyère Research Institute and Centre de recherche (Ottawa) and Institut universitaire de gériatrie de Montréal (Montréal). Available: https://deprescribing.org/resources/deprescribing -guidelines-algorithms (accessed 2019 Mar. 3).

32. 2019 American Geriatrics Society Beers Criteria ${ }^{\circledR}$ Update Expert Panel. American Geriatrics Society 2019 Updated AGS Beers Criteria ${ }^{\circledR}$ for potentially inappropriate medication use in older adults. $7 \mathrm{Am}$ Geriatr Soc 2019;67:674-94.

33. Morgan SG, Hunt J, Rioux J, et al. Frequency and cost of potentially inappropriate prescribing for older adults: a cross-sectional study. CMAZ Open 2016;4:E346-51.

34. Black CD, Thavorn K, Coyle D, et al. The health system costs of potentially inappropriate prescribing: a population-based, retrospective cohort study using linked health administrative databases in Ontario, Canada. Pharmacoeconon Open 2020;4:27-36.

35. Tadrous M, Martins D, Mamdani MM, et al. Characteristics of high-drug-cost beneficiaries of public drug plans in 9 Canadian provinces: a cross-sectional analysis. CMA7 Open 2020;8:E297-303.

36. Hill AB. The Environment and Disease: Association or Causation? 7 R Soc Med. 1965;58(5):295-300. doi:10.1177/003591576505800503

37. Table 17-10-0005-01: Population estimates on July 1st, by age and sex. Ottawa: Statistics Canada; modified 2020 Sept. 14. Available: www150.statcan.gc.ca/t1/ tbl1/en/tv.action?pid=1710000501 (accessed 2019 Sept. 15).
38. Juurlink D, Preyra C, Croxford R, et al. Canadian Institute for Health Information Discharge Abstract Database: a validation study [ICES investigative report]. Toronto; ICES: 2006. Available: www.ices.on.ca/Publications/ Atlases-and-Reports/2006/Canadian-Institute-for-Health-Information (accessed 2019 Oct. 1).

Affiliations: Division of Geriatric Medicine (Lee), Department of Medicine, and Department of Health Research Methods, Evidence, and Impact (Lee, Muratov, Tarride, Mbuagbaw, Seow, Thabane, Holbrook), and Centre for Health Economics and Policy Analysis (CHEPA) (Tarride), McMaster University, Hamilton, Ont.; ICES (Paterson, Gomes, Khuu, Seow, Thavorn); Institute of Health Policy, Management and Evaluation (Paterson, Thavorn), University of Toronto, Toronto, Ont.; Ottawa Hospital Research Institute (Thavorn), The Ottawa Hospital, Ottawa, Ont.; Li Ka Shing Knowledge Institute (Gomes), St. Michael's Hospital, Toronto, Ont.; Department of Oncology (Seow), Faculty of Health Sciences, and Division of Clinical Pharmacology and Toxicology (Holbrook), Department of Medicine, McMaster University, Hamilton, Ont.

Contributors: Justin Lee, Anne Holbrook, Sergei Muratov, Jean-Eric Tarride, J. Paterson and Kednapa Thavorn conceptualized the study. All authors contributed to its design. Justin Lee drafted the initial protocol manuscript. All authors gave final approval of the version to be published and agreed to be accountable for all aspects of the work.

Funding: This work is supported by the Ontario Drug Policy Research Network which is funded by grants from the Ontario Ministry of Health and Long-Term Care, and the Ontario Strategy for Patient-Oriented Research Support Unit, which is supported by CIHR and the Province of Ontario. This study will also be supported by ICES, which is funded by an annual grant from the Ontario Ministry of Health and Long-Term Care, and by personnel funding from the Canadian Institutes of Health Research (CIHR) Fellowship Program, the CIHR Drug Safety and Effectiveness Cross-Disciplinary Training Program, The Research Institute of St. Joe's Hamilton and the St. Peter's/McMaster Chair in Aging.

Content licence: This is an Open Access article distributed in accordance with the terms of the Creative Commons Attribution (CC BYNCND 4.0) licence, which permits use, distribution and reproduction in any medium, provided that the original publication is properly cited, the use is noncommercial (i.e., research or educational use), and no modifications or adaptations are made. See: https://creativecommons.org/ licenses/by-nc-nd/4.0/

Data sharing: The data set from this study will be held securely in coded form at ICES. Although data-sharing agreements prohibit ICES from making the data set publicly available, access may be granted to those who meet pre-specified criteria for confidential access, available at www.ices.on. $\mathrm{ca} / \mathrm{DAS}$. The full data set creation plan and underlying analytic code will be available from the authors on request, with the understanding that the computer programs may rely on coding templates or macros that are unique to ICES and are therefore inaccessible or may require modification.

Supplemental information: For reviewer comments and the original submission of this manuscript, please see www.cmajopen.ca/content/9/1/ E44/suppl/DC1.

Disclaimer: This study is supported by ICES, which is funded by an annual grant from the Ontario Ministry of Health and Long-Term Care (MOHLTC). The opinions, results and conclusions reported in this article are those of the authors and are independent from the funding sources. No endorsement by ICES or the Ontario MOHLTC is intended or should be inferred. 